\title{
Robust Redundant Input Reliable Tracking Control for Omnidirectional Rehabilitative Training Walker
}

\author{
Ping Sun, ${ }^{1}$ Shuoyu Wang, ${ }^{2}$ and Hamid Reza Karimi ${ }^{3}$ \\ ${ }^{1}$ School of Information Science and Engineering, Shenyang University of Technology, Shenyang 110870, China \\ ${ }^{2}$ Department of Intelligent Mechanical Systems Engineering, Kochi University of Technology, Kochi 7828502, Japan \\ ${ }^{3}$ Department of Engineering, Faculty of Engineering and Science, University of Agder, 4898 Grimstad, Norway
}

Correspondence should be addressed to Hamid Reza Karimi; hamid.r.karimi@uia.no

Received 21 October 2013; Revised 16 December 2013; Accepted 17 December 2013; Published 30 January 2014

Academic Editor: Xiaojie Su

Copyright (c) 2014 Ping Sun et al. This is an open access article distributed under the Creative Commons Attribution License, which permits unrestricted use, distribution, and reproduction in any medium, provided the original work is properly cited.

\begin{abstract}
The problem of robust reliable tracking control on the omnidirectional rehabilitative training walker is examined. The new nonlinear redundant input method is proposed when one wheel actuator fault occurs. The aim of the study is to design an asymptotically stable controller that can guarantee the safety of the user and ensure tracking on a training path planned by a physical therapist. The redundant degrees of freedom safety control and the asymptotically zero state detectable concept of the walker are presented, the model of redundant degree is constructed, and the property of center of gravity constant shift is obtained. A controller that can satisfy asymptotic stability is obtained using a common Lyapunov function for admissible uncertainties resulting from an actuator fault. Simulation results confirm the effectiveness of the proposed method and verify that the walker can provide safe sequential motion when one wheel actuator is at fault.
\end{abstract}

\section{Introduction}

Modern control applications are characterized by the presence of multiple actuators inducing the same effects on the plant dynamics. It is a natural way to introduce redundancy into a control scheme for enhancing reliability. However, how do we realize reliability using these redundant actuators? When the actuator fault has been detected, nonfault functioning actuators can maintain the sequential working of a system that normally operates with redundant actuators. Then, what are the changes of the system performance? Clearly, these problems are fundamentally important in modern control theory. Considering theoretical study and engineering applications simultaneously, there are many problems worth studying. This paper will start a certain extent research on omnidirectional rehabilitative training walker.

Omnidirectional walker (ODW) is a class of typical wheeled mobile robots that have redundant actuators. That can help patients undergoing rehabilitation training move in any direction on a flat surface and can be programmed to follow specific training trajectories that make up recovery training programs for various illnesses and injuries. Image of the ODW is presented in Figure 1.
The effectiveness of ODW in rehabilitation has been verified through clinical tests $[1,2]$, which has motivated research into various aspects of ODW performance. The work in [3] studied the problem of robust control using a kinematic equation; however, the kinetic equation was not analyzed. Consequently, the analysis could not account for patients with different weights. The work in $[4,5]$ researched tracking control on mobile robots using a kinematic equation. It was found that tracking neglects the mass and inertia matrix when the loads are overweight, and consequently tracking precision is reduced. An adaptive control method for the ODW was discussed in [6-8]. The kinetic equation was improved by considering the center of gravity shifts and load fluctuations caused by users. The adaptive controller obtained favorable tracking. However, many parameters needed to be adjusted in the controller. To operate effectively in real-world applications, the control algorithm must guarantee that they can follow a prescribed path accurately.

Extensive results about tracking control and nonlinear control have been reported in literature. For example, tracking control for stochastic modeling was studied in $[9,10]$, fuzzy logic tracking control for autonomous mobile robot was 


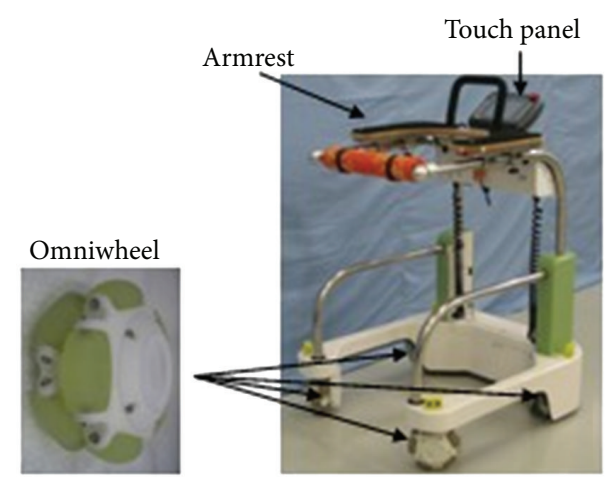

FIGURE 1: Omnidirectional walker and omniwheel.

investigated in [11], dynamic output feedback for nonlinear systems was addressed in $[12,13]$, fuzzy control was studied in [14], and dissipativity-based sliding mode control was studied in [15]. Note that all the abovementioned results were obtained under the assumption that the actuator motors are fault-free. However, in practical applications, actuator failures are often unavoidable. Therefore, to guarantee performance safety, the controller must be reliable [16, 17]. To ensure system reliability, many modern control applications, particularly in aircraft and robotic systems, are characterized by redundant actuators $[18,19]$.

From previous studies, we know that the ODW has a redundant actuator degree of freedom [20]. In [20], the problem of center of gravity which does not shift was solved in tracking control. Actuators are very important in transferring the controller output to the plant. Therefore, determining how to maintain both stability and a bound of a certain cost in the presence of actuator failures is a worthwhile endeavor. Previous research assumed that actuators never fail and can reliably stabilize wheeled mobile robots, including the ODW. This assumption ignores safety issues. To the best of the authors' knowledge, the corresponding problems on safety of the redundant input system when actuators fail have not been investigated yet, while research in this area is clearly very important from both theoretical and practical points of view, which motivate us to carry out the present work.

In this study, the main contributions of this paper can be summarized as follows.

(i) The redundant input is very important when one actuator fails. In this study, a redundant input kinetic model is constructed by separating the corresponding columns of the control matrix.

(ii) The property of center of gravity constant shift is obtained. The controller is gotten for admissible control actuator failures; that is, the resulting design could tolerate the actuator failure and maintain the stabizlity of the system by constructing a common Lyapunov function.

(iii) Reliability goals are intended to ensure system performance and are not exclusively related to safety. When an actuator fails, the corresponding column of

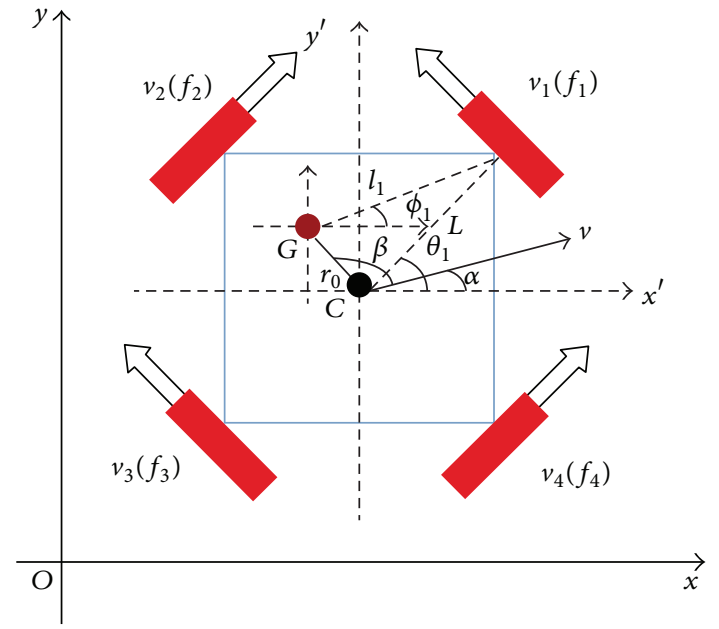

FIGURE 2: Structure of omnidirectional walker.

the control matrix will be separated from the original control matrix. The structure of system will be changed. Therefore, maintaining system tracking performance is very important. The sufficient condition of tracking error performance is derived for admissible uncertainties that an actuator fault causes.

(iv) As an application, the robust reliable tracking control on the ODW is considered. On the basis of the redundant input model, the efficiency of the proposed scheme is demonstrated. The proposed method can be extended to solve safety problems in other wheeled mobile robots.

The remainder of this paper is organized as follows. In Section 2, the model of the ODW with redundant degree of freedom is formulated. The main results that provide a solution to the robust reliable tracking control problem are presented in Section 3. Simulation results are given in Section 4 , and concluding remarks are provided in Section 5.

Notation. The following notations are used throughout the paper: for a vector $x \in R^{k}$, the 2-norm of $x$ is defined as $|x|=\left(x^{T} x\right)^{1 / 2}$; for a matrix $X, X^{-1}$ denotes its inverse and $X^{T}$ denotes its transpose; for a matrix $Q, \lambda_{\max }(Q)$ and $\lambda_{\text {min }}(Q)$ are defined as the maximum eigenvalue and the minimum eigenvalue of $Q$, respectively. I denotes the identity matrix with an appropriate dimension.

\section{Redundant Input Model of the Omnidirectional Walker}

The coordinate settings and structure used to develop the safety control for the ODW are shown in Figure 2. We will deduce the redundant input model on the basis of the kinetic equation in [21].

In Figure 2,

$$
\begin{aligned}
& \Sigma(x, O, y): \text { absolute coordinate system } \\
& \Sigma\left(x^{\prime}, C, y^{\prime}\right): \text { translation coordinate system }
\end{aligned}
$$


$v$ : speed of the ODW

$v_{i}$ : speed of an omniwheel

$f_{i}$ : force on each omniwheel

$G$ : center of gravity of the walker

$r_{0}$ : distance between $G$ and the center of gravity due to the load

$\alpha$ : angle between the $x^{\prime}$ axis and the direction of $v$

$\beta$ : angle between the $x^{\prime}$ axis and $r_{0}$

$L$ : distance from the center of the ODW to each omniwheel

$l_{i}$ : distance from the center of gravity to the middle of each omniwheel

$\theta_{i}$ : angle between the $x^{\prime}$ axis and the position of each omniwheel

$\phi_{i}$ : angle between the $x^{\prime}$ axis and $l_{i}$.

The kinetic model is expressed as

$$
M_{0} K \ddot{X}+M_{0} \dot{K} \dot{X}=K_{G}^{T} F,
$$

where

$$
\begin{aligned}
& M_{0}=\left[\begin{array}{ccc}
M+m & 0 & 0 \\
0 & M+m & 0 \\
0 & 0 & I_{0}+m r_{0}^{2}
\end{array}\right], \quad K=\left[\begin{array}{lll}
1 & 0 & p \\
0 & 1 & q \\
0 & 0 & 1
\end{array}\right], \\
& X=\left[\begin{array}{c}
x_{C} \\
y_{C} \\
\theta
\end{array}\right], \quad K_{G}^{T}=\left[\begin{array}{cccc}
-\sin \theta_{1} & \sin \theta_{2} & \sin \theta_{3} & -\sin \theta_{4} \\
\cos \theta_{1} & -\cos \theta_{2} & \cos \theta_{3} & \cos \theta_{4} \\
\lambda_{1} & -\lambda_{2} & -\lambda_{3} & \lambda_{4}
\end{array}\right] \text {, } \\
& \lambda_{1}=l_{1} \cos \left(\theta_{1}-\phi_{1}\right) \text {, } \\
& \lambda_{2}=l_{2} \cos \left(\theta_{2}-\phi_{2}\right) \text {, } \\
& \lambda_{3}=l_{3} \cos \left(\theta_{3}-\phi_{3}\right) \text {, } \\
& \lambda_{4}=l_{4} \cos \left(\theta_{4}-\phi_{4}\right) \text {, }
\end{aligned}
$$

$$
\begin{gathered}
p=\frac{1}{2}\left[\left(\lambda_{1}-\lambda_{3}\right) \sin \theta+\left(\lambda_{2}-\lambda_{4}\right) \cos \theta\right], \\
q=\frac{1}{2}\left[\left(\lambda_{2}-\lambda_{4}\right) \sin \theta-\left(\lambda_{1}-\lambda_{3}\right) \cos \theta\right], \\
F=\left[\begin{array}{l}
f_{1} \\
f_{2} \\
f_{3} \\
f_{4}
\end{array}\right],
\end{gathered}
$$

where $M$ is the mass of the ODW, $m$ is the user's equivalent mass, which varies according to the user's weight and walking disability, and $I_{0}$ is the inertia of mass of the walker. $m r_{0}^{2}$ is the inertia of mass cause by $m . f_{1}, f_{2}, f_{3}$, and $f_{4}$ are the input forces of the system; $\ddot{x}_{c}, \ddot{y}_{c}$, and $\ddot{\theta}$ are the output of the system; $m$ and $r_{0}$ are variable parameters.

As can be seen from the differential equations (1), the system is nonlinear because the direction angle $\theta$ changes over time. $\theta$ is the angle between the $x^{\prime}$ axis and the position of the first omniwheel, $\theta=\theta_{1}$, then we have $\theta_{2}=\theta+\pi / 2$, $\theta_{3}=\theta+\pi$, and $\theta_{4}=\theta+3 \pi / 2$. Although four control input forces are found, $f_{1}, f_{2}, f_{3}$, and $f_{4}$, only three are independent. This implies that the walker has a redundant degree of freedom.

The problem of center of gravity constant shift will be solved in this paper. From the expression of $p$, $q$, we can obtain

$$
\begin{gathered}
\dot{p}=-q \dot{\theta} \\
\dot{q}=p \dot{\theta}
\end{gathered}
$$

To deal with the problem of center of gravity constant shift, using matrix $\left(M_{0} K\right)^{T}$ premultiplication (1). The kinetic model can be expressed as

$$
M_{1} \ddot{X}+M_{2} \dot{X}=B(\theta) F,
$$

where

$$
\begin{aligned}
& M_{1}=\left[\begin{array}{ccc}
(M+m)^{2} & 0 & p(M+m)^{2} \\
0 & (M+m)^{2} & q(M+m)^{2} \\
p(M+m)^{2} & q(M+m)^{2} & \left(I_{0}+m r_{0}^{2}\right)^{2}+\frac{(M+m)^{2}}{4}\left[\left(\lambda_{1}-\lambda_{3}\right)^{2}+\left(\lambda_{2}-\lambda_{4}\right)^{2}\right]
\end{array}\right] \\
& M_{2}=\left[\begin{array}{ccc}
0 & 0 & -q \dot{\theta}(M+m)^{2} \\
0 & 0 & p \dot{\theta}(M+m)^{2} \\
0 & 0 & 0
\end{array}\right] \\
& B(\theta)=\left[\begin{array}{cccc}
-(M+m) \sin \theta & (M+m) \cos \theta & -(M+m) \sin \theta & (M+m) \cos \theta \\
(M+m) \cos \theta & (M+m) \sin \theta & -(M+m) \cos \theta & (M+m) \sin \theta \\
\lambda_{31} & \lambda_{32} & \lambda_{33} & \lambda_{34}
\end{array}\right] \\
& \lambda_{31}=-(M+m) p \sin \theta+(M+m) q \cos \theta+\lambda_{1}\left(I_{0}+m r_{0}^{2}\right) \text {, } \\
& \lambda_{32}=(M+m) p \cos \theta+(M+m) q \sin \theta-\lambda_{2}\left(I_{0}+m r_{0}^{2}\right) \text {, }
\end{aligned}
$$




$$
\begin{gathered}
\lambda_{33}=-(M+m) p \sin \theta-(M+m) q \cos \theta-\lambda_{3}\left(I_{0}+m r_{0}^{2}\right), \\
\lambda_{34}=(M+m) p \cos \theta+(M+m) q \sin \theta+\lambda_{4}\left(I_{0}+m r_{0}^{2}\right) .
\end{gathered}
$$

Definition 1. For a nonlinear system

$$
\dot{x}(t)=f(x, t)+B u(t) .
$$

For control matrix $B \in R^{n \times m}$, if $\operatorname{rank}(B)=\min \{n, m\}$, then $B$ has redundant degree of $d(d=\max \{n, m\}-\min \{n, m\})$. Control actuators with less $d$ are at fault; the other actuators can sustain regular motion of the system (6); we can state the system can realize safety using redundant degree of freedom. Furthermore, if the system (6) can track the prescribed path sequentially when control actuators of less $d$ are at fault, we can say the system (6) can guarantee tracking cost.

Remark 2. In fact, the control matrix $B$ has a nullspace of $d$ dimension in which $u(t)$ can be perturbed without affecting the system dynamic. These redundant actuators are not functional when all redundant actuators are fault-free. However, if some actuators of less $d$ are at fault, the redundant actuators are very important to maintain safe sequential motion. Many modern control applications have redundant actuators to promote system safety.

The problem of safety control in the presence of actuator failures that may occur in the control channels is considered. The walker can realize safety reliable tracking control through its redundant degree of freedom. The fault actuator input force will be separated from (4) to obtain the following redundant input model:

$$
M_{1} \ddot{X}+M_{2} \dot{X}=B_{0}(\theta) F_{0}+\Delta B_{0}(\theta) \Delta F_{0} .
$$

Define $B_{0}(\theta) F_{0}=u(t), \Delta B_{0}(\theta) \Delta F_{0}=\omega(t)$. So

$$
M_{1} \ddot{X}+M_{2} \dot{X}=u(t)+\omega(t)
$$

where $F_{0}$ denotes the normal control input force of the system. $\Delta F_{0}$ is regarded as extrinsic bounded interference that represents the input force of actuator failure and satisfies

$$
-\left|f_{i}\right|_{\max } \leq \Delta F_{0}(t) \leq\left|f_{i}\right|_{\max }, \quad i=1,2,3,4 .
$$

Remark 3. Many modern control applications are characterized by multiple actuators. Modern aircrafts, especially military planes, often use redundant actuators to improve maneuverability and reliability [22]. The ODW has a redundant actuator that is not important when the ODW is in normal motion; however, it is very important to guarantee the safety of the ODW when an actuator is at fault. Therefore, construction of the redundant model (8) becomes very important.

Remark 4. As in [23], the generalized force $\tau$ can be decomposed as the dissipative force, the control force, and the random excitation force. In this study, the control input
$F$ in (4) can be decomposed as $F_{0}$ and $\Delta F_{0}$ in (7). $F_{0}$ is caused by the control input acting on the system, and $\Delta F_{0}$ is caused by the control input of an actuator failure. It is worth noting that $\Delta F_{0}$ will produce the uncertain term of $\Delta B_{0}(\theta) \Delta F_{0}$. It is well known that mechanical systems are often subject to uncertainty, which can significantly affect system performance $[24,25]$. The purpose of this study is to solve the ODW safety problem under the uncertainty $\Delta B_{0}(\theta) \Delta F_{0}$. When an actuator fails during the course of system operation, $\Delta B_{0}(\theta)$ is the corresponding column of the control matrix $B(\theta)$ and regards uncertainty extrinsic bounded interference.

The desired motion trajectory is $X_{d}$, and the actual motion trajectory is $X$; therefore, tracking error $e(t)$ is

$$
\begin{aligned}
& e(t)=X_{d}-X, \\
& \ddot{e}(t)=\ddot{X}_{d}-\ddot{X},
\end{aligned}
$$

where

$$
e(t)=\left(\begin{array}{l}
e_{1}(t) \\
e_{2}(t) \\
e_{3}(t)
\end{array}\right)=\left(\begin{array}{l}
x_{d}-x \\
y_{d}-y \\
\theta_{d}-\theta
\end{array}\right), \quad E(t)=\dot{e}(t)
$$

The error state equation is

$$
M_{1} \ddot{e}+M_{2} \dot{e}=M_{1} \ddot{X}_{d}+M_{2} \dot{X}_{d}-u(t)-\omega(t) .
$$

This paper is to design a robust controller for the system (12) such that, for center of gravity constant shift and extrinsic bounded interference $\Delta B_{0}(\theta) \Delta F_{0}$, the following two requirements are simultaneously satisfied.

(C1) The error system (12) is asymptotically stable.

(C2) Given $\gamma>0, \rho_{1}>0$ and $\rho_{2}>0$, the tracking error performance satisfies

$$
\int_{0}^{\infty}\|\dot{e}(t)\|_{2}^{2} d t \leq \rho_{1} V(0)+\rho_{2} \int_{0}^{\infty}\|\omega(t)\|_{2}^{2} d t .
$$

Remark 5. This study examines the problem of tracking control. Tracking error is an important performance index. Therefore, the guaranteed tracking cost is given as (13) such that the tracking error $e(t)$ is restricted an upper bounded when the structure of the system (4) is changed as a result of actuator failure. This approach (13) has the advantage of providing the upper bound on a given performance index, and thus the system performance degradation incurred by the model bounded interference is guaranteed to be less than this bound. 


\section{The Design of the Robust Controller}

Our purpose is to design a safety controller that can track the paths defined in walking training programs to ensure compliance with the rehabilitation regime when one wheel actuator fault occurs and center of gravity constant shifts. The robust tracking control for the error state equation (12) is stated as follows.

Definition 6. If there exist control laws $u(t)$, such that for all admissible actuator failures, the error state equation (12) is asymptotically stable and the corresponding tracking error function (13) is satisfied, then $u(t)$ is said to be a guaranteed safety robust reliable control law.

Remark 7. The safety control can be realized when the actuator fault has been detected. Therefore, three nonfault functioning actuators can maintain the programmed sequential motion of a walker that normally operates with four actuators. The corresponding fault column was separated from the control matrix $B(\theta)$ and regarded as extrinsic bounded interference. At the same time, the initial input forces must increase to compensate for the tracking error. This condition is dangerous for both the walker and trainer. The functioning actuators will be damaged by the disproportionate input forces, and the walker will lose stability. Here, robust reliable control and the tracking error function have been proposed to address this issue.

Definition 8 . The ODW error tracking system

$$
\begin{gathered}
M_{1} \ddot{e}+M_{2} \dot{e}=M_{1} \ddot{X}_{d}+M_{2} \dot{X}_{d}-u(t)-\omega(t), \\
y(t)=e(t)
\end{gathered}
$$

is called asymptotically zero state detectable if for any $\varepsilon>0$, there exists $\delta(\varepsilon)>0$, such that when $|y(t+s)|<\delta$ holds for some $t \geq 0, h>0$, and $0 \leq s<h$, we have $\|E(t)\|<\varepsilon$.

Remark 9. Definition 8 is the asymptotic zero state detectability concept. Detectability is a useful property when addressing asymptotic stability. Here, asymptotic zero state detectability is a limited-time norm observability.

Proposition 10. The center of gravity is constant shift for the $O D W$, and $M+m=C, C$ is a constant. Then, $\dot{M}_{1}=M_{2}+M_{2}^{T}$.

Proof. The center of gravity is constant shift, which is the center of gravity that gets to a fixed point. So, $\lambda_{i}, i=1,2,3,4$ is a constant. When $M+m=C$, we obtain

$$
\dot{M}_{1}=\left[\begin{array}{ccc}
0 & 0 & -q \dot{\theta}(M+m)^{2} \\
0 & 0 & p \dot{\theta}(M+m)^{2} \\
-q \dot{\theta}(M+m)^{2} & p \dot{\theta}(M+m)^{2} & 0
\end{array}\right] .
$$

From the expression of $M_{2}$, we complete the proof of Proposition 10.

Remark 11. The center of gravity is constant shift that is a general phenomenon due to the load changes for Wheeled Mobile Robot, including the ODW. This phenomenon causes that the ODW cannot accurately follow a training path planned by a physical therapist for walking rehabilitation. Maybe, the ODW will bump into an obstacle, so, it is dangerous for the user. Proposition 10 is an important conclusion for solving the problem of center of gravity constant shift.

Next, we design control laws for the system (12) by employing a common Lyapunov function technique to solve the problem of guaranteed safety control for admissible actuator failures.

Theorem 12. Considering the redundant input error state equation (12), suppose that there exist scalar symmetric matrices $P>0, Q>0$, positive constants $\gamma>0, \rho_{1}>0$ and $\rho_{2}>0$, and that the system of (12) is asymptotically zero state detectable. Then, the control input (16)

$$
\begin{gathered}
u(t)=u_{f}+\frac{M_{2}+M_{2}^{T}}{2} \dot{e}(t), \\
u_{f}=M_{1} \ddot{X}_{d}+M_{2} \dot{X}_{d}-\left(M_{2}-\frac{1}{2 \gamma^{2}} I\right) \dot{e}(t)+Q \dot{e}(t)+P e(t)
\end{gathered}
$$

solves the problem of robust reliable tracking control for actuator failures.

Moreover, the tracking error performance satisfies

$$
\int_{0}^{\infty}\|\dot{e}(t)\|_{2}^{2} d t \leq \rho_{1} V(0)+\rho_{2} \int_{0}^{\infty}\|\omega(t)\|_{2}^{2} d t .
$$

Further, the control input forces are given by

$$
\begin{aligned}
F_{0}=B_{0}^{-1}(\theta)[ & M_{1} \ddot{X}_{d}+M_{2} \dot{X}_{d}-\left(M_{2}-\frac{1}{2 \gamma^{2}} I\right) \dot{e}(t) \\
& \left.+Q \dot{e}(t)+P e(t)+\frac{M_{2}+M_{2}^{T}}{2} \dot{e}(t)\right] .
\end{aligned}
$$

Proof. $M_{1}$ is a positive symmetric matrix. Define the Lyapunov function

$$
V(t)=\frac{1}{2} \dot{e}^{T}(t) M_{1} \dot{e}(t)+\frac{1}{2} e^{T}(t) P e(t) .
$$

The time derivative of $V(t)$ along the trajectory of system (12) is given by

$$
\begin{aligned}
\dot{V}(t)= & \dot{e}^{T}(t) M_{1} \ddot{e}(t)+\frac{1}{2} \dot{e}^{T}(t) \dot{M}_{1} \dot{e}(t)+\dot{e}^{T}(t) P e(t) \\
= & \dot{e}^{T}(t)\left[M_{1} \ddot{X}_{d}+M_{2} \dot{X}_{d}-M_{2} \dot{e}(t)-u(t)-\omega(t)\right] \\
& +\dot{e}^{T}(t) P e(t)+\frac{1}{2} \dot{e}^{T}(t) \dot{M}_{1} \dot{e}(t) \\
= & \dot{e}^{T}(t)\left[M_{1} \ddot{X}_{d}+M_{2} \dot{X}_{d}-M_{2} \dot{e}(t)-u_{f}+P e(t)\right] \\
& -\dot{e}^{T}(t) \frac{M_{2}+M_{2}^{T}}{2} \dot{e}(t)+\frac{1}{2} \dot{e}^{T}(t) \dot{M}_{1} \dot{e}(t)-\dot{e}^{T}(t) \omega(t)
\end{aligned}
$$




$$
\begin{aligned}
= & \dot{e}^{T}(t)\left[M_{1} \ddot{X}_{d}+M_{2} \dot{X}_{d}\right. \\
& \left.-\left(M_{2}-\frac{1}{2 \gamma^{2}} I\right) \dot{e}(t)-u_{f}+P e(t)\right] \\
- & \frac{1}{2}\left(\frac{1}{\gamma} \dot{e}(t)+\gamma \omega(t)\right)^{T}\left(\frac{1}{\gamma} \dot{e}(t)+\gamma \omega(t)\right) \\
+ & \frac{1}{2} \gamma^{2} \omega^{T}(t) \omega(t) \\
\leq & \dot{e}^{T}(t)\left[M_{1} \ddot{X}_{d}+M_{2} \dot{X}_{d}\right. \\
& \left.\quad-\left(M_{2}-\frac{1}{2 \gamma^{2}} I\right) \dot{e}(t)-u_{f}+P e(t)\right] \\
+ & \frac{1}{2} \gamma^{2} \omega^{T}(t) \omega(t) .
\end{aligned}
$$

From (16) and Proposition 10

$$
\dot{V}(t) \leq-\dot{e}^{T}(t) Q \dot{e}(t)+\frac{1}{2} \gamma^{2} \omega^{T}(t) \omega(t) .
$$

Choosing $\lambda_{\min }(Q)>(1 / 2) \gamma^{2} \omega^{2}(t)$, we have

$$
\dot{V}(t)<0 \text {. }
$$

Thus, according to the asymptotical zero state detectability, the error state equation (12) is asymptotically stable.

In the following, we show that the system satisfies the tracking error performance. Furthermore, integrate the inequalities (21) from 0 to $\infty$ on both sides, it follows

$$
\begin{gathered}
\int_{0}^{\infty} \dot{V}(t) d t \leq \int_{0}^{\infty}-\dot{e}^{T}(t) Q \dot{e}(t) d t+\frac{1}{2} \int_{0}^{\infty} \gamma^{2} \omega^{T}(t) \omega(t) d t \\
-V(0) \leq-\int_{0}^{\infty} \dot{e}^{T}(t) Q \dot{e}(t) d t+\frac{1}{2} \int_{0}^{\infty} \gamma^{2} \omega^{T}(t) \omega(t) d t \\
\int_{0}^{\infty} \dot{e}^{T}(t) Q \dot{e}(t) d t \leq V(0)+\frac{1}{2} \int_{0}^{\infty} \gamma^{2} \omega^{T}(t) \omega(t) d t \\
\int_{0}^{\infty} \lambda_{\min }(Q)\|\dot{e}(t)\|_{2}^{2} d t \leq V(0)+\frac{1}{2} \gamma^{2} \int_{0}^{\infty} \omega^{T}(t) \omega(t) d t \\
\int_{0}^{\infty}\|\dot{e}(t)\|_{2}^{2} d t \leq \frac{1}{\lambda_{\min }(Q)} V(0) \\
+\frac{1}{2 \lambda_{\min }(Q)} \gamma^{2} \int_{0}^{\infty} \omega^{T}(t) \omega(t) d t
\end{gathered}
$$

Let $\rho_{1}=1 / \lambda_{\min }(Q), \rho_{2}=\gamma^{2} / 2 \lambda_{\min }(Q)$; therefore

$$
\int_{0}^{\infty}\|\dot{e}(t)\|_{2}^{2} d t \leq \rho_{1} V(0)+\rho_{2} \int_{0}^{\infty}\|\omega(t)\|_{2}^{2} d t
$$

is the tracking error performance upper bound of system (12). This completes the proof of Theorem 12.
Remark 13. The safety reliable controller is easily solved by a common Lyapunov function, and the tracking error performance upper bound, required to prevent the initial oversize input forces caused by the initial tracking error, can be obtained from $V(t)$. We propose a method that is much easier to satisfy than the partial differential inequalities in [26]. In addition, reliable control for linear systems against actuator faults by the introduction of an adaptive mechanism has been researched in $[27,28]$; fault values must be estimated online and adjust control law to maintain satisfactory performances.

Remark 14. A new method against actuator faults has been proposed by separating the corresponding columns of the control matrix based on redundant degree of freedom. The fault values must not be estimated online compared with general reliable controller design, and reliable tracking control can be realized by others nonfault functioning actuators.

\section{The Simulation Results}

In this section, the proposed robust redundant input reliable tracking control algorithm is verified by the ODW linear path tracking simulation.

Consider actuators that may fail during the course of system operation. Suppose that for any $t$, only one actuator fails. Without loss of generality, we consider that the fourth actuator is at fault. Thus, the error state system (12) can be rewritten as

$$
M_{1} \ddot{e}+M_{2} \dot{e}=M_{1} \ddot{X}_{d}+M_{2} \dot{X}_{d}-u(t)-\omega(t),
$$

where $u(t)=B_{0}(\theta) F_{0}, \omega(t)=\Delta B_{0}(\theta) \Delta F_{0}$, and

$$
\begin{gathered}
B_{0}(\theta)=\left[\begin{array}{ccc}
-(M+m) \sin \theta & (M+m) \cos \theta & -(M+m) \sin \theta \\
(M+m) \cos \theta & (M+m) \sin \theta & -(M+m) \cos \theta \\
\lambda_{31} & \lambda_{32} & \lambda_{33}
\end{array}\right], \\
\Delta B_{0}(\theta)=\left[\begin{array}{c}
(M+m) \cos \theta \\
(M+m) \sin \theta \\
\lambda_{34}
\end{array}\right], \quad F_{0}=\left[\begin{array}{c}
f_{1} \\
f_{2} \\
f_{3}
\end{array}\right], \\
\Delta F_{0}=f_{4} .
\end{gathered}
$$

In a typical rehabilitation scenario, the ODW must follow a predefined path that consists of a series of linear paths. Here, to verify the tracking performance of the proposed method rigorously, we assume that the walker follows a linear path. The reference trajectory $X_{d}$ is described by

$$
\begin{gathered}
x_{d}(t)=20\left(1-e^{-0.1 t}\right), \\
y_{d}(t)=20\left(1-e^{-0.1 t}\right), \\
\theta_{d}(t)=\frac{\pi}{2} .
\end{gathered}
$$

The physical parameters of the ODW used in the simulation are $M=58 \mathrm{~kg}, L=0.4 \mathrm{~m}, \gamma=0.1, I_{0}=27.7 \mathrm{~kg} \cdot \mathrm{m}^{2}$, and load $m=60 \mathrm{~kg}$. The centers of constant shift are $r_{0}=0.1 \mathrm{~m}$, and $\beta=\pi / 4$. 


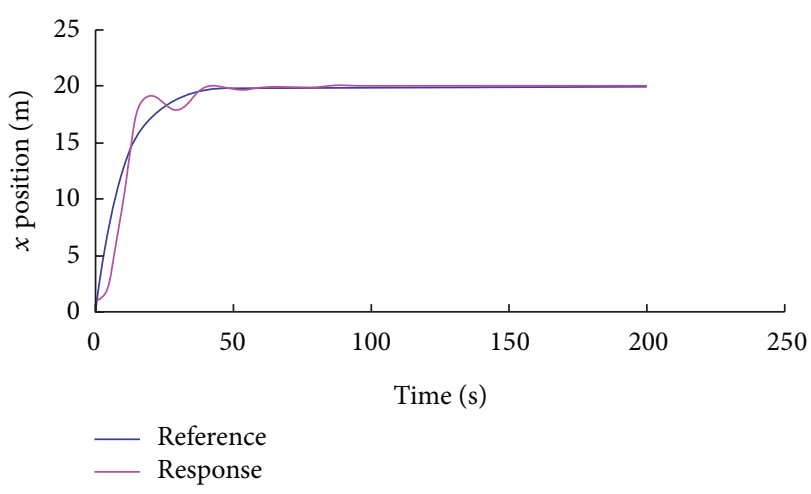

FIGURE 3: Trajectory tracking of $x$ position.

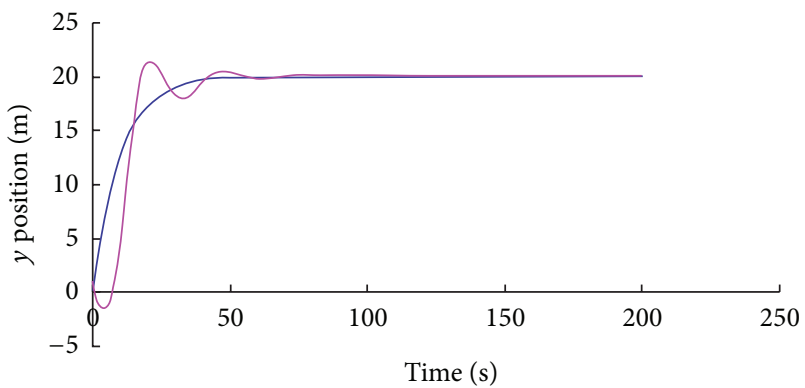

— Reference

FIGURE 4: Trajectory tracking of $y$ position.

As an example, we consider the stuck failure of the fourth actuator, $f_{4}=1 \mathrm{~N}$. The ODW has extrinsic bounded

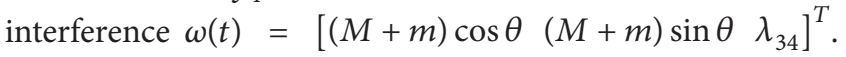
The controller parameters are $P=\operatorname{diag}\{1000,800,1000\}$ and $Q=\operatorname{diag}\{1800,1900,1800\}$. The simulation results are given in the following figures.

Figures 3, 4, and 5 plot the trajectories of the $x$ and $y$ positions and the orientation angle, respectively. The error state equation (12) can realize asymptotic stability in limited time. The input forces are shown in Figure 6. The other actuators input energies converge to neighborhood of zero when one actuator is stuck in failure. So, three nonfault functioning actuators can maintain the programmed sequential motion of the ODW that normally operates with four actuators and restrain extrinsic bounded interference $\omega(t)$ resulting from the fourth actuator fault. The reliable control method can guarantee the walker's continuous safe motion when one actuator fails.

To verify the effectiveness of redundant input reliable control to deal with actuator failures, we conducted comparative simulations with the four input forces controller. If all the actuators are normal, the redundant input method is not used to decompose the kinetic model (4); the error state equation is

$$
M_{1} \ddot{e}+M_{2} \dot{e}=M_{1} \ddot{X}_{d}+M_{2} \dot{X}_{d}-u(t),
$$

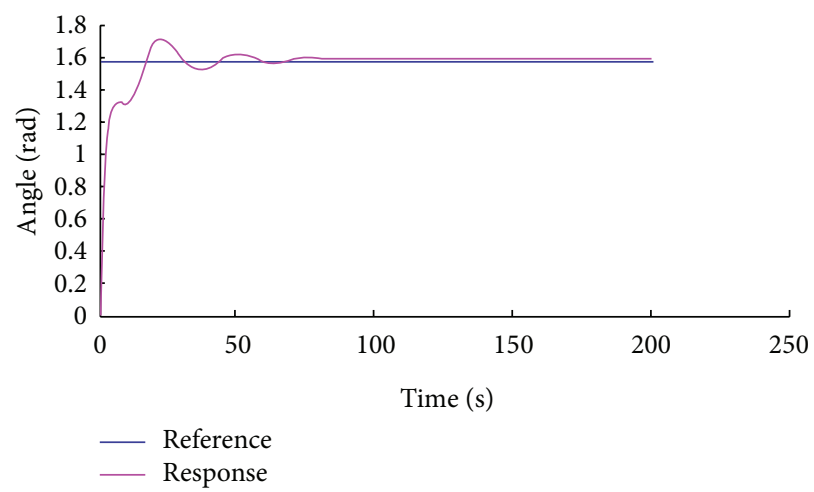

FIGURE 5: Trajectory tracking of angle.

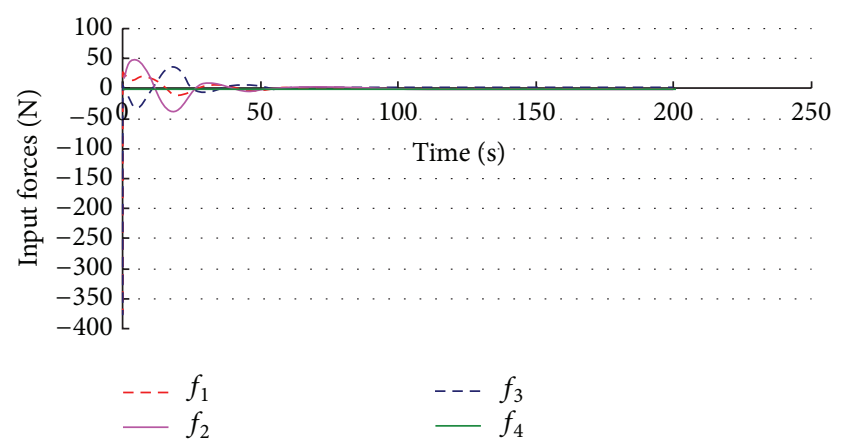

FIGURE 6: Input forces.

where $u(t)=B(\theta) F$, using the same Lyapunov function $V(t)$, and the four control input forces $f_{i}$ can be expressed as follows:

$$
\begin{aligned}
& F=\widehat{B}(\theta)[ M_{1} \ddot{X}_{d}+M_{2} \dot{X}_{d}-M_{2} \dot{e}(t) \\
&\left.+Q \dot{e}(t)+P e(t)+\frac{M_{2}+M_{2}^{T}}{2} \dot{e}(t)\right], \\
& \widehat{B}(\theta)=B^{T}(\theta)\left(B(\theta) B^{T}(\theta)\right)^{-1},
\end{aligned}
$$

where $\widehat{B}(\theta)$ is the pseudoinverse of control matrix $B(\theta)$ in model (4).

Using the controller (29), and while all of the parameters do not change, the walker can follow the specified training trajectory $X_{d}$. The simulation results are given in the following figures.

Figures 7, 8, and 9 plot the tracking performance of the ODW for the $x$ position, $y$ position, and orientation angle, respectively. Figure 10 plots the input forces of the ODW. It is evident that the walker can realize asymptotic stability and trajectory tracking. These simulation results demonstrate that the controller (29) is only effective when all the actuators are fault-free. However, one wheel actuator $f_{4}$ fails abruptly when the ODW is in motion; the walker must rely on the remaining three functioning actuators to maintain the training sequence. The results of the comparative simulation are presented in the following figures. 


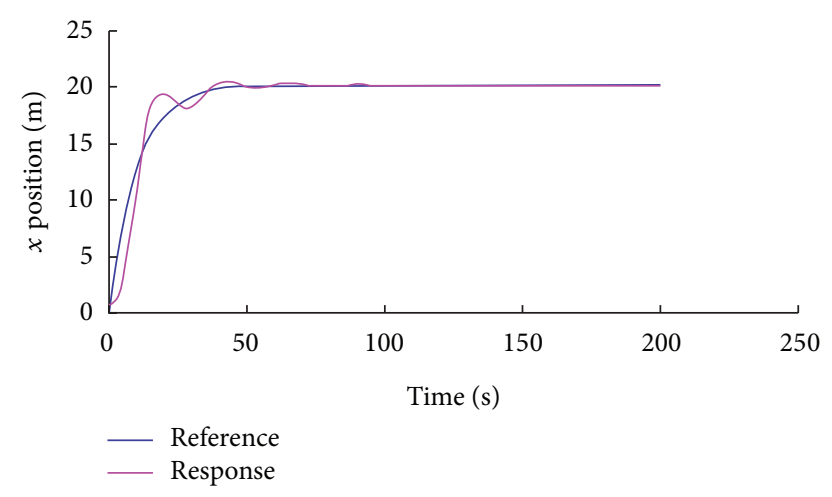

FIGURE 7: Trajectory tracking of $x$ position.

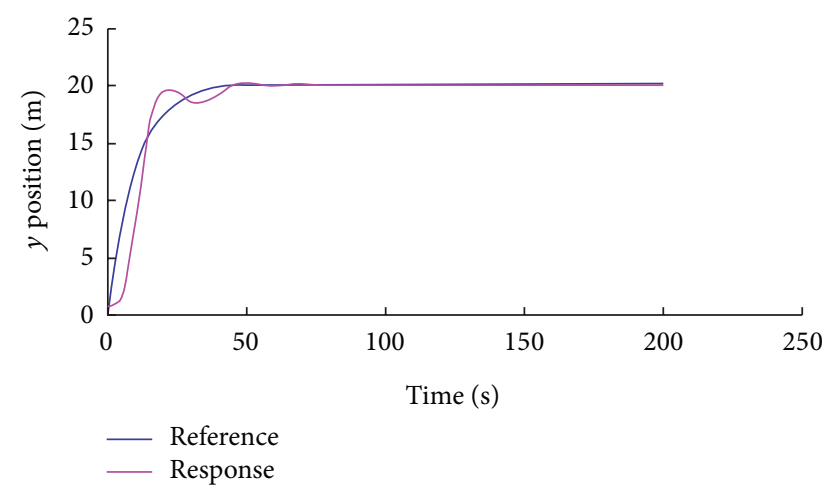

FIGURE 8: Trajectory tracking of $y$ position.

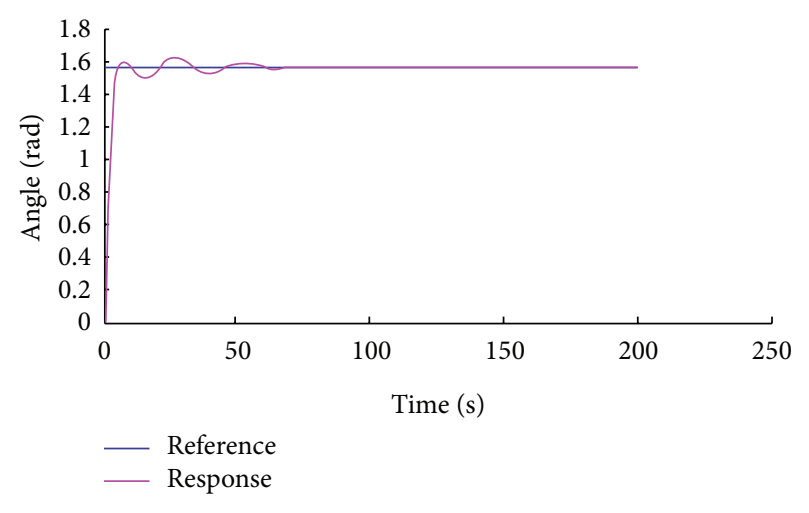

Figure 9: Trajectory tracking of angle.

As shown in Figures 11, 12, and 13, after approximately $70 \mathrm{~s}$, the ODW can track training trajectory of $x$ position; the tracking error is larger than that in Figure 3. However, the ODW cannot track training trajectory of $y$ position and orientation angle; the error state equation (12) cannot realize asymptotic stability. Figure 14 shows the input forces fluctuate partly in the first $70 \mathrm{~s}$ because the ODW do not stabilize. In particular, the user may be in danger because the actual path is far from the desired path in Figures 12 and 13. Given such dramatic deviation from the desired path, the ODW could bump into an obstacle and potentially even be a danger to

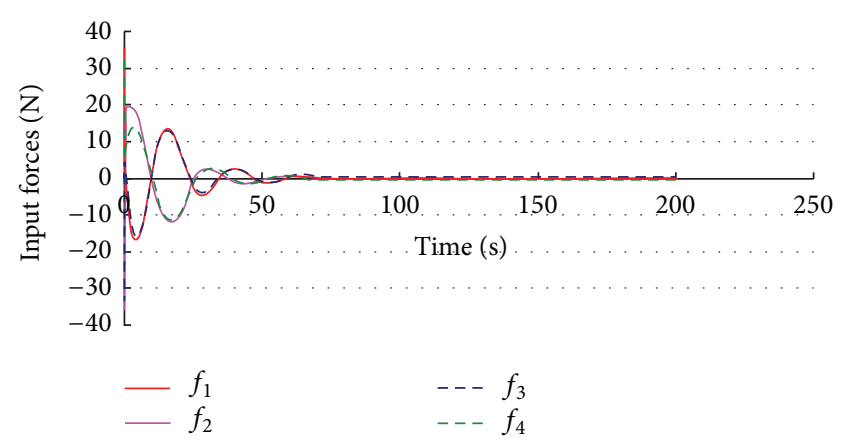

FIGURE 10: Input forces.

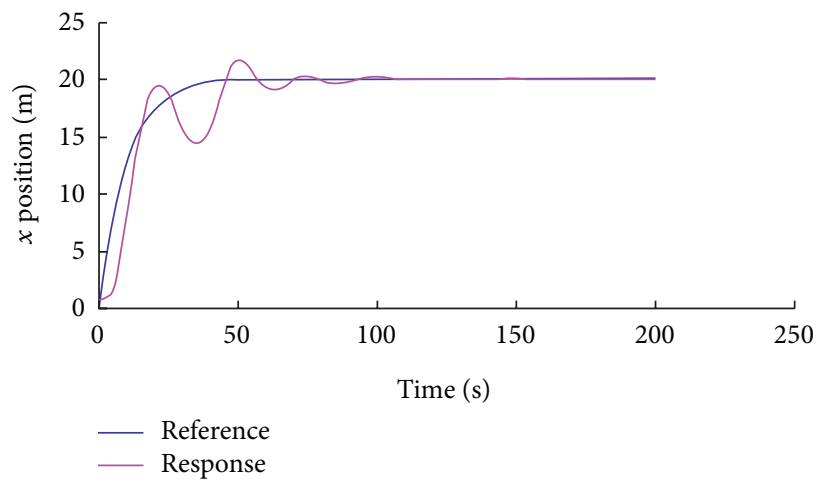

FIGURE 11: Trajectory tracking of $x$ position.

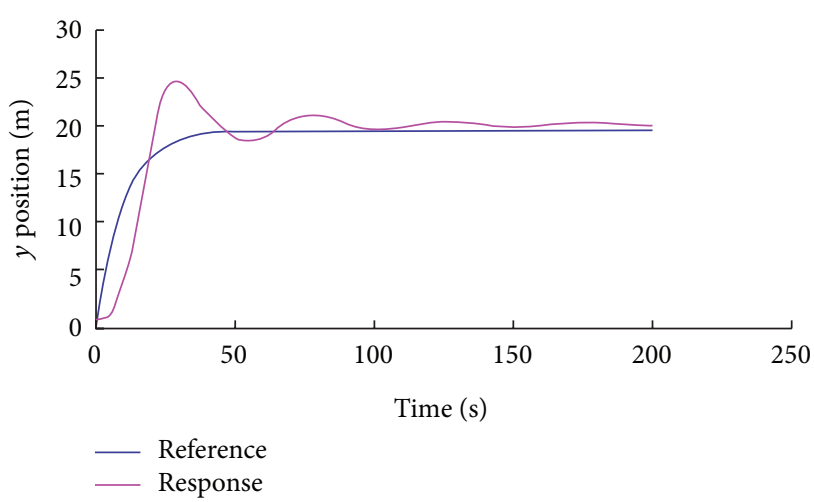

FIGURE 12: Trajectory tracking of $y$ position.

others in the rehabilitation facility because of the unstable input forces in Figure 14.

We developed the safety mechanism to ensure that patients would have the best opportunity to regain normal walking ability through a variety of ODW training programs. The redundant input reliable control method exhibits good tracking performance for a training path planned by a physical therapist when one actuator fault occurs and the center of gravity is constant shift, and the user is safe even though a wheel actuator fails. Therefore, according to Definition 1, the proposed control method, which was verified by simulations, can ensure patient safety and satisfy the tracking performance. 


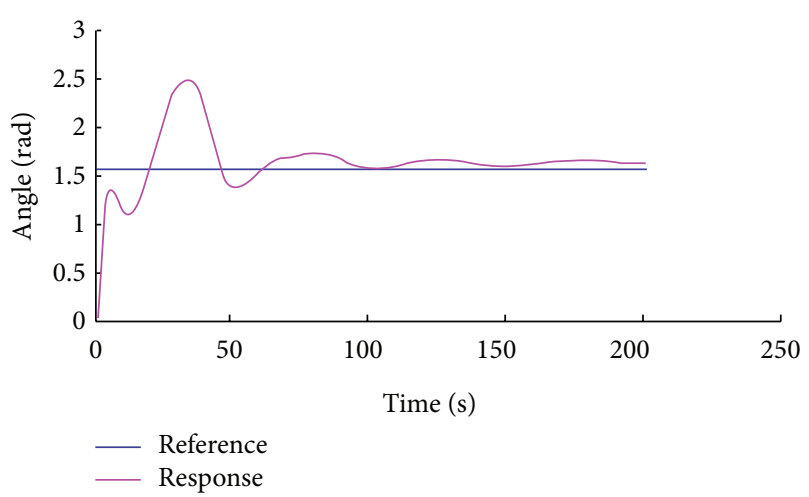

FIgURE 13: Trajectory tracking of angle.

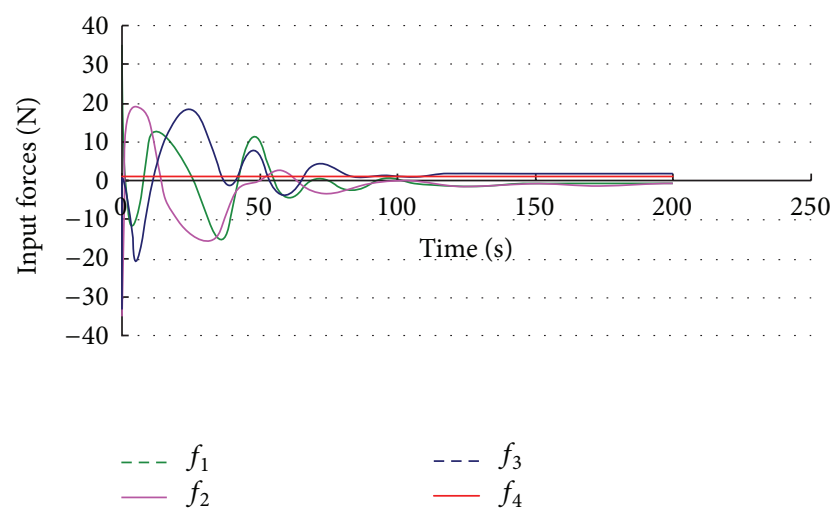

FIGURE 14: Input forces.

\section{Conclusions}

Guaranteeing the safety of patients using the ODW when an actuator fault and the center of gravity constant shift occur is very important. The nonlinear robust redundant input reliable control method is proposed. By using the common Lyapunov function, the obtained safety controller can stabilize the walker. The tracking results are consistent with a preprogrammed training path designed by a medical professional. The proposed method focuses on actuator failures, which have not been addressed in previous studies. Simulation results for a new synthesis design to resolve safety issues associated with actuator failures have demonstrated the effectiveness of the proposed method. It is probable that, besides the ODW, the proposed method can also be applied to other wheeled mobile robots.

The robust reliable controller can track the path planned by a physical therapist when one wheel actuator is at fault based on the assumptions that the center of gravity is constant shift. However, the position of the center of gravity is usually time varying during the walking training. To address this issue, further work will focus on developing a new control method.

\section{Conflict of Interests}

The authors declare that there is no conflict of interests regarding the publication of this paper.

\section{Acknowledgments}

The authors would like to thank the Associate Editor and the reviewers for their constructive comments and suggestions that have helped improve the quality and presentation of this paper. This study was supported by the Japan Society for the Program of Science KAKENHI Grant nos. 24300203, 23240088, and 22300197, and the Canon Foundation.

\section{References}

[1] S. Y. Wang, H. Inoue, and K. Kawata, "Developing the omnidirectional mobile walker and verifying its effect of increase in the muscle power," in Proceedings of the JSME Symposium on Walfare Engineering, pp. 176-177, 2007.

[2] K. Ishida, S. Y. Wang, and T. Nagano, "Development of an allway mobile walker," Journal of Physical Medicine, vol. 19, no. 4, pp. 246-250, 2008 (Japanese).

[3] J. Yang, X. Chen, H. Guo, and Q. Zhang, "Implementation of omnidirectional lower limbs rehabilitation training robot," in Proceedings of the International Conference on Electrical Machines and Systems (ICEMS '07), pp. 2033-2036, Seoul, Republic of Korea, October 2007.

[4] S. X. Yang, A. Zhu, G. Yuan, and M. Q.-H. Meng, "A bioinspired neurodynamics-based approach to tracking control of mobile robots," IEEE Transactions on Industrial Electronics, vol. 59, no. 8, pp. 3211-3220, 2012.

[5] D. K. Chwa, "Fuzzy adaptive tracking control of wheeled mobile robots with state-dependent kinematic and dynamic disturbances," IEEE Transactions on Fuzzy Systems, vol. 20, no. 3, pp. 587-593, 2012.

[6] R. Tan, S. Wang, Y. Jiang, K. Ishida, and M. Nagano, "Path tracking control considering center of gravity shift and load change for an omni-directional walker," in Proceedings of the IEEE International Conference on Information and Automation (ICIA '10), pp. 672-675, Harbin, China, June 2010.

[7] R. Tan, S. Wang, Y. Jiang, K. Ishida, M. G. Fujie, and M. Nagano, "Adaptive control method for path-tracking control of an omnidirectional walker compensating for center-of-gravity shifts and load changes," International Journal of Innovative Computing, Information and Control, vol. 7, no. 7, pp. 4423-4434, 2011.

[8] R. Tan, S. Wang, Y. Jiang, K. Ishida, and M. Nagano, "Adaptive controller for omni-directional walker," in Proceedings of the International Conference on Mechatronics and Automation (ICMA '10), pp. 156-161, Xian, China, August 2010.

[9] H. Q. Wang, B. Chen, and C. Lin, "Adaptive neural tracking control for a class of stochastic nonlinear systems with unknown dead-zone," International Journal of Innovative Computing, Information and Control, vol. 9, no. 8, pp. 3257-3269, 2013.

[10] M. Y. Cui, Z. J. Wu, and X. J. Xie, "Stochastic modeling and tracking control for a two-link planar rigid robot manipulator," International Journal of Innovative Computing, Information and Control, vol. 9, no. 4, pp. 1769-1780, 2013.

[11] L. Astudillo, P. Melin, and O. Castillo, "Chemical optimization paradigm applied to a fuzzy tracking controller for an autonomous mobile robot," International Journal of Innovative 
Computing, Information and Control, vol. 9, no. 5, pp. 20072018, 2013.

[12] L. G. Wu, X. J. Su, and P. Shi, "Output feedback control of Markovian jump repeated scalar nonlinear systems," IEEE Transaction on Automatic Control, vol. 58, no. 1, pp. 1-6, 2013.

[13] X. J. Su, P. Shi, L. G. Wu, and Y.-D. Song, "A novel control design on discrete-time Takagi-Sugeno fuzzy systems with time-varying delays," IEEE Transaction on Fuzzy Systems, vol. 21, no. 4, pp. 655-671, 2013.

[14] X. J. Su, X. Z. Yang, P. Shi, and L. G. Wu, "Fuzzy control of nonlinear electromagnetic suspension systems," Mechatronics, 2013.

[15] L. G. Wu, W. X. Zheng, and H. J. Guo, "Dissipativity-based sliding mode control of switched stochastic systems," IEEE Transaction on Automatic Control, vol. 58, no. 3, pp. 785-791, 2013.

[16] G.-H. Yang, S.-Y. Zhang, J. Lam, and J. Wang, "Reliable control using redundant controllers," IEEE Transactions on Automatic Control, vol. 43, no. 11, pp. 1588-1593, 1998.

[17] O. Härkegård and S. T. Glad, "Resolving actuator redundancy optimal control versus control allocation," Automatica, vol. 41, no. 1, pp. 137-144, 2005.

[18] L. Zaccarian, "On dynamic control allocation for input-redundant control systems," in Proceedings of the 46th IEEE Conference on Decision and Control (CDC '07), pp. 1192-1197, New Orleans, La, USA, December 2007.

[19] Z. Duan, L. Huang, and Y. Yang, "The effects of redundant control inputs in optimal control," Science in China F, vol. 52, no. 11, pp. 1973-1981, 2009.

[20] P. Sun and S. Y. Wang, "Robust input redundant reliable tracking control for omni-directional rehabilitative training walker," ICIC Express Letters, vol. 8, no. 1, pp. 79-85, 2014.

[21] R. Tan, S. Wang, Y. Jiang, K. Ishida, T. Chai, and M. G. Fujie, "Adaptive controller for omni-directional walker: improvement of dynamic model," in Proceedings of the International Conference on Mechatronics and Automation (ICMA '11), pp. 325-330, Beijing, China, August 2011.

[22] Z. Duan, L. Huang, Y. Yao, and Z.-P. Jiang, "On the effects of redundant control inputs," Automatica, vol. 48, no. 9, pp. 21682174, 2012.

[23] M.-Y. Cui, Z.-J. Wu, X.-J. Xie, and P. Shi, "Modeling and adaptive tracking for a class of stochastic lagrangian control systems," Automatica, vol. 49, no. 3, pp. 770-779, 2013.

[24] Q. Zhou, P. Shi, J. Lu, and S. Xu, "Adaptive output-feedback fuzzy tracking control for a class of nonlinear systems," IEEE Transactions on Fuzzy Systems, vol. 19, no. 5, pp. 972-982, 2011.

[25] Y. Yin, P. Shi, and F. Liu, "Gain-scheduled PI tracking control on stochastic nonlinear systems with partially known transition probabilities," Journal of the Franklin Institute, vol. 348, no. 4, pp. 685-702, 2011.

[26] R. Wang and J. Zhao, "Reliable guaranteed cost control for uncertain switched non-linear systems," International Journal of Systems Science, vol. 40, no. 3, pp. 205-211, 2009.

[27] G.-H. Yang and D. Ye, "Reliable $H_{\infty}$ control of linear systems with adaptive mechanism," IEEE Transactions on Automatic Control, vol. 55, no. 1, pp. 242-247, 2010.

[28] D. Ye and G.-H. Yang, "Reliable guaranteed cost control for linear state delayed systems with adaptive memory state feedback controllers," Asian Journal of Control, vol. 10, no. 6, pp. 678-686, 2008. 


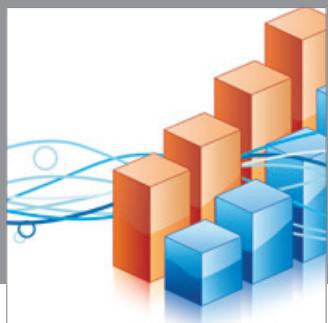

Advances in

Operations Research

mansans

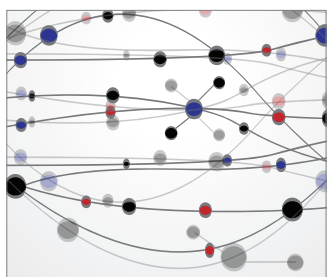

The Scientific World Journal
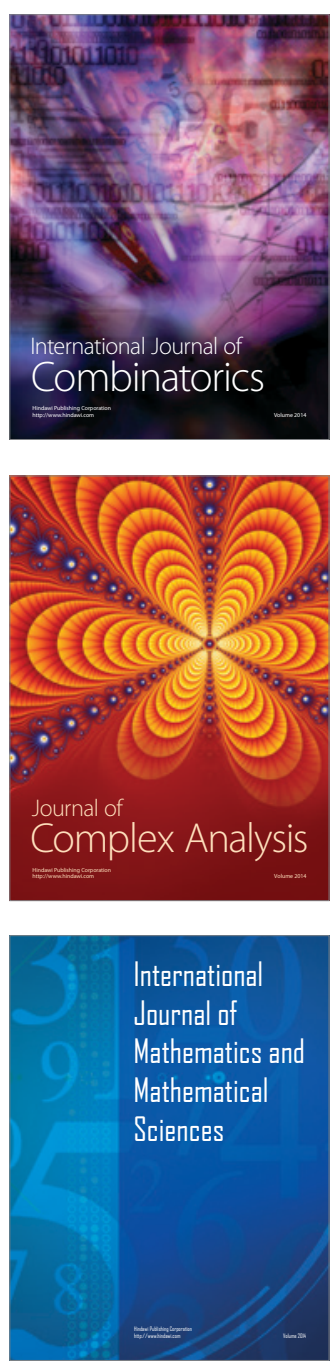
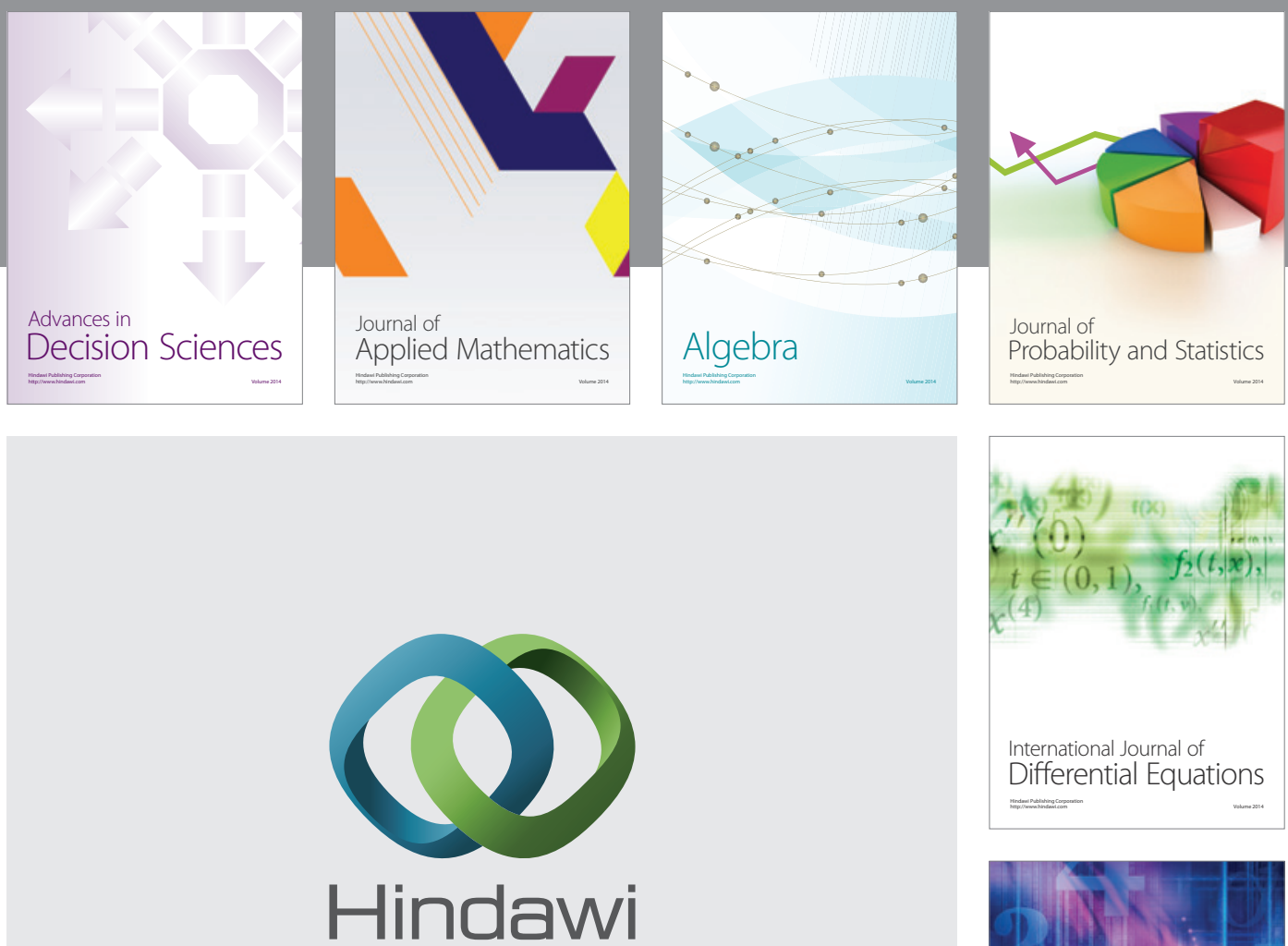

Submit your manuscripts at http://www.hindawi.com
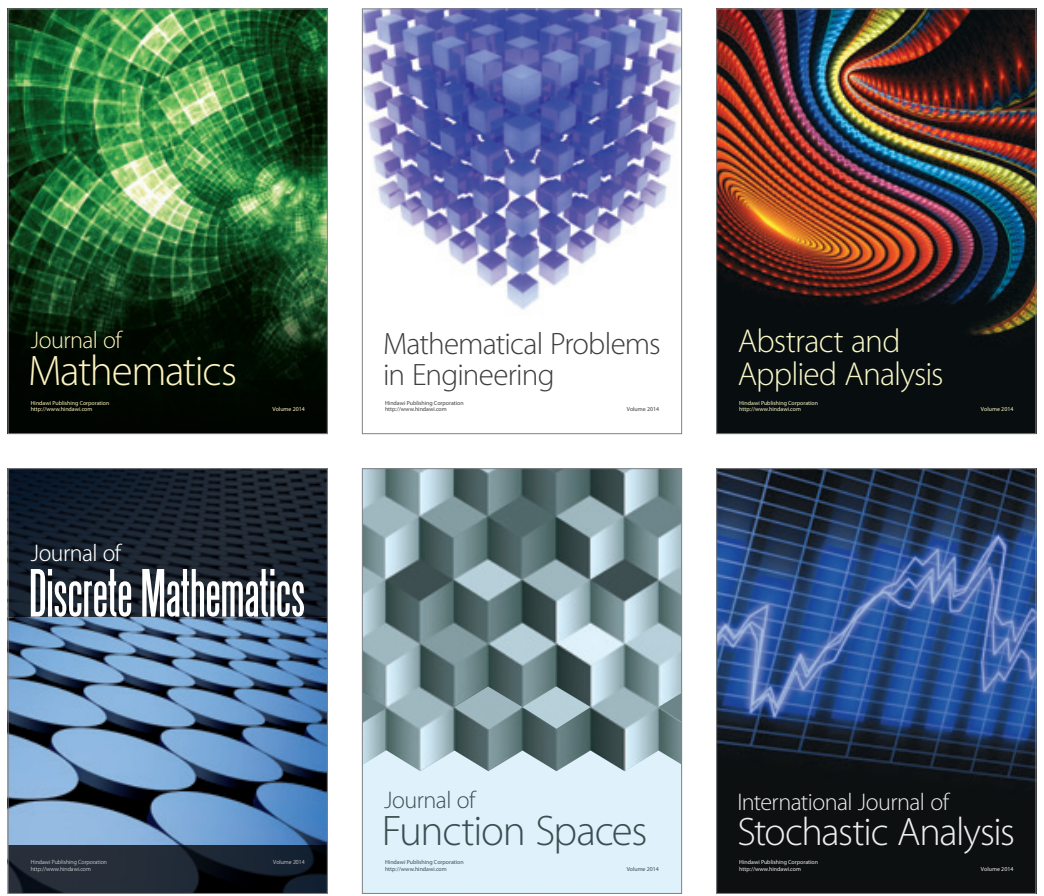

Journal of

Function Spaces

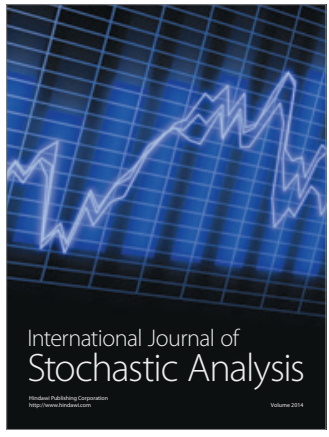

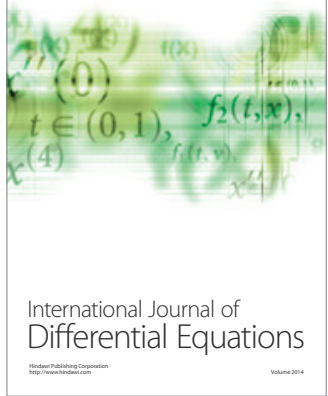
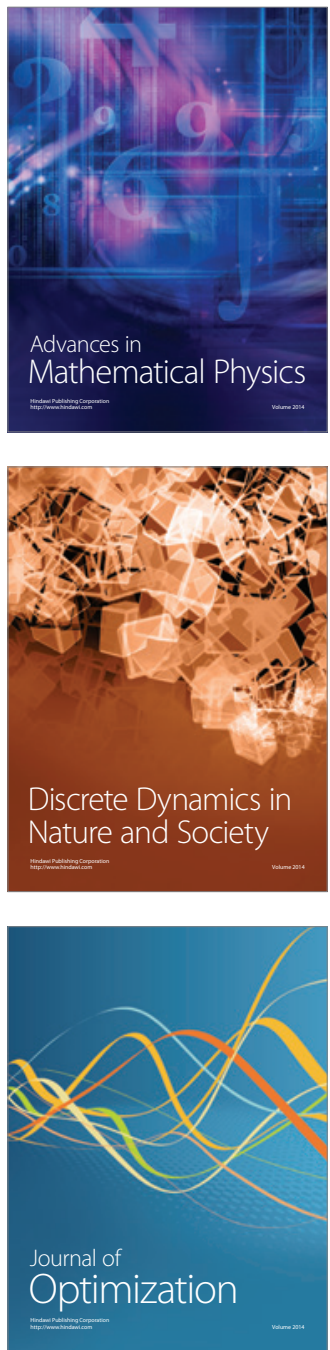